\title{
Gap Junction
}

National Cancer Institute

\section{Source}

National Cancer Institute. Gap Junction. NCI Thesaurus. Code C13312.

Intercellular connections comprised of complexes of connexin family proteins that permit the transfer of cytoplasmic ions and molecules between connected cells. Each cell contains a hemichannel made up of homo- or hetero-hexamers of connexins. 